densed neutron phase would start at the center. By reason of the greater density of the condensed phase, the star will begin to collapse. The details of this process are difficult to analyze without knowing the change of density and the heat of condensation (latent heat of evaporation). If the latter one can be neglected beside the regular energy liberation in the stellar interior, collapsing can go on until a very thin neutron atmosphere is left around the condensed neutron core. This hypothesis affords a concrete physical basis for Zwicky's ${ }^{6}$ suggestion that the supernovae originate from the sudden transition of an ordinary star to a centrally condensed one. It is obvious that a detailed analysis of this problem must await a great deal more experimental data concerning the physical properties of the neutron.

I should like to express my thanks to Dr. Rupert Wildt for helpful discussions on the subject.

${ }^{6}$ F. Zwicky, Astrophys. J. 88, 522 (1938).

\title{
On Continued Gravitational Contraction
}

\author{
J. R. Oppenheimer and H. SNyder \\ University of California, Berkeley, California
}

(Received July 10, 1939)

\begin{abstract}
When all thermonuclear sources of energy are exhausted a sufficiently heavy star will collapse. Unless fission due to rotation, the radiation of mass, or the blowing off of mass by radiation, reduce the star's mass to the order of that of the sun, this contraction will continue indefinitely. In the present paper we study the solutions of the gravitational field equations which describe this process. In I, general and qualitative arguments are given on the behavior of the metrical tensor as the contraction progresses: the radius of the star approaches asymptotically its gravitational radius; light from the surface of the star is progressively reddened, and can escape over a progressively narrower range of angles. In II, an analytic solution of the field equations confirming these general arguments is obtained for the case that the pressure within the star can be neglected. The total time of collapse for an observer comoving with the stellar matter is finite, and for this idealized case and typical stellar masses, of the order of a day; an external observer sees the star asymptotically shrinking to its gravitational radius.
\end{abstract}

\section{I}

$\mathrm{R}^{\mathrm{E}}$ ECENTLY it has been shown ${ }^{1}$ that the general relativistic field equations do not possess any static solutions for a spherical distribution of cold neutrons if the total mass of the neutrons is greater than $\sim 0.7 \odot$. It seems of interest to investigate the behavior of nonstatic solutions of the field equations.

In this work we will be concerned with stars which have large masses, $>0.7 \odot$, and which have used up their nuclear sources of energy. A star under these circumstances would collapse under the influence of its gravitational field and release energy. This energy could be divided into four parts: (1) kinetic energy of motion of the

${ }^{1}$ J. R. Oppenheimer and G. M. Volkoff, Phys. Rev. 55, 374 (1939). particles in the star, (2) radiation, (3) potential and kinetic energy of the outer layers of the star which could be blown away by the radiation, (4) rotational energy which could divide the star into two or more parts. If the mass of the original star were sufficiently small, or if enough of the star could be blown from the surface by radiation, or lost directly in radiation, or if the angular momentum of the star were great enough to split it into small fragments, then the remaining matter could form a stable static distribution, a white dwarf star. We consider the case where this cannot happen.

If then, for the late stages of contraction, we can neglect the gravitational effect of any escaping radiation or matter, and may still neglect the deviations from spherical symmetry 
produced by rotation, the line element outside the boundary $r_{b}$ of the stellar matter must take the form

$$
d s^{2}=e^{\nu} d t^{2}-e^{\lambda} d r^{2}-r^{2}\left(d \theta^{2}+\sin ^{2} \theta d \varphi^{2}\right)
$$

with

$$
e^{\nu}=\left(1-r_{0} / r\right)
$$

and

$$
e^{\lambda}=\left(1-r_{0} / r\right)^{-1} \text {. }
$$

Here $r_{0}$ is the gravitational radius, connected with the gravitational mass $m$ of the star by $r_{0}=2 m g / c^{2}$, and constant. We should now expect that since the pressure of the stellar matter is insufficient to support it against its own gravitational attraction, the star will contract, and its boundary $r_{b}$ will necessarily approach the gravitational radius $r_{0}$. Near the surface of the star, where the pressure must in any case be low, we should expect to have a local observer see matter falling inward with a velocity very close to that of light ; to a distant observer this motion will be slowed up by a factor $\left(1-r_{0} / r_{b}\right)$. All energy emitted outward from the surface of the star will be reduced very much in escaping, by the Doppler effect from the receding source, by the large gravitational red-shift, $\left(1-r_{0} / r_{b}\right)^{\frac{1}{2}}$, and by the gravitational deflection of light which will prevent the escape of radiation except through a cone about the outward normal of progressively shrinking aperture as the star contracts. The star thus tends to close itself off from any communication with a distant observer; only its gravitational field persists. We shall see later that although it takes, from the point of view of a distant observer, an infinite time for this asymptotic isolation to be established, for an observer comoving with the stellar matter this time is finite and may be quite short.

Inside the star we shall still suppose that the matter is spherically distributed. We may then take the line element in the form (1). For this line element the field equations are

$$
\begin{aligned}
-8 \pi T_{1}{ }^{1} & =e^{-\lambda}\left(\nu^{\prime} / r+1 / r^{2}\right)-1 / r^{2}, \\
8 \pi T_{4}{ }^{4} & =e^{-\lambda}\left(\lambda^{\prime} / r-1 / r^{2}\right)+1 / r^{2}, \\
-8 \pi T_{2}{ }^{2} & =-8 \pi T_{3}{ }^{3} \\
& =e^{-\lambda}\left(\frac{\nu^{\prime \prime}}{2}+\frac{\nu^{\prime 2}}{4}-\frac{\nu^{\prime} \lambda^{\prime}}{4}+\frac{\nu^{\prime}-\lambda^{\prime}}{2 r}\right) \\
& -e^{-\nu}\left(\ddot{\lambda} / 2+\dot{\lambda}^{2} / 4-\dot{\lambda} \dot{\nu} / 4\right), \\
8 \pi T_{4}{ }^{1} & =-8 \pi e^{\nu-\lambda} T_{1}{ }^{4}=-e^{-\lambda \dot{\lambda} / r} ;
\end{aligned}
$$

in which primes represent differentiation with respect to $r$ and dots differentiation with respect to $t$.

The energy-momentum tensor $T_{\nu}^{\mu}$ is composed of two parts: (1) a material part due to electrons, protons, neutrons and other nuclei, (2) radiation. The material part may be thought of as that of a fluid which is moving in a radial direction, and which in comoving coordinates would have a definite relation between the pressure, density, and temperature. The radiation may be considered to be in equilibrium with the matter at this temperature, except for a flow of radiation due to a temperature gradient.

We have been unable to integrate these equations except when we place the pressure equal to zero. However, one can obtain some information about the solutions from inequalities implied by the differential equations and from conditions for regularity of the solutions. From Eqs. (2) and (3) one can see that unless $\lambda$ vanishes at least as rapidly as $r^{2}$ when $r \rightarrow 0, T_{4}^{4}$ will become singular and that either or both $T_{1}^{1}$ and $\nu^{\prime}$ will become singular. Physically such a singularity would mean that the expression used for the energy-momentum tensor does not take account of some essential physical fact which would really smooth the singularity out. Further, a star in its early stage of development would not possess a singular density or pressure; it is impossible for a singularity to develop in a finite time.

If, therefore, $\lambda(r=0)=0$, we can express $\lambda$ in terms of $T_{4}^{4}$, for, integrating Eq. (3)

$$
\lambda=-\ln \left\{1-\frac{8 \pi}{r} \int_{0}^{r} T_{4}^{4} r^{2} d r\right\} .
$$

Therefore $\lambda \geqslant 0$ for all $r$ since $T_{4}^{4} \geqslant 0$.

Now that we know $\lambda \geqslant 0$, it is easy to obtain some information about $\nu^{\prime}$ from Eq. (2);

$$
\nu^{\prime} \geqslant 0
$$

since $\lambda$ and $-T_{1}{ }^{1}$ are equal to or greater than zero.

If we use clock time at $r=\infty$, we may take $\nu(r=\infty)=0$. From this boundary condition and Eq. (7) we deduce

$$
\nu \leqslant 0 .
$$

The condition that space be flat for large $r$ is 
$\lambda(r=\infty)=0$. Adding Eqs. (2) and (3) we obtain :

$$
8 \pi\left(T_{4}^{4}-T_{1}^{1}\right)=e^{\lambda}\left(\lambda^{\prime}+\nu^{\prime}\right) / r .
$$

Since $T_{4}{ }^{4}$ is greater than zero and $T_{1}{ }^{1}$ is less than zero we conclude

$$
\lambda^{\prime}+\nu^{\prime} \geqslant 0
$$

Because of the boundary conditions on $\lambda$ and $\nu$ we have

$$
\lambda+\nu \leqslant 0 .
$$

For those parts of the star which are collapsing, i.e., all parts of the star except those being blown away by the radiation, Eq. (5) tells us that $\dot{\lambda}$ is greater than zero. Since $\lambda$ increases with time, it may (a) approach an asymptotic value uniformly as a function of $r$; or (b) increase indefinitely, although certainly not uniformly as a function of $r$, since $\lambda(r=0)=0$. If $\lambda$ were to approach a limiting value the star would be approaching a stationary state. However, we are supposing that the relationships between the $T_{\nu}{ }^{\mu}$ do not admit any stationary solutions, and therefore exclude this possibility. Under case (b) we might expect that for any value of $r$ greater than zero, $\lambda$ will become greater than any preassigned value if $t$ is sufficiently large. If this were so the volume of the star

$$
V=4 \pi \int_{0}^{r_{b}} e^{\lambda / 2} r^{2} d r
$$

would increase indefinitely with time; since the mass is constant, the mean density in the star would tend to zero. We shall see, however, that for all values of $r$ except $r_{0}, \lambda$ approaches a finite limiting value; only for $r=r_{0}$ does it increase indefinitely.

\section{II}

To investigate this question we will solve the field equations with the limiting form of the energy-momentum tensor in which the pressure is zero. When the pressure vanishes there are no static solutions to the field equations except when all components of $T_{\nu}{ }^{\mu}$ vanish. With $p=0$ we have the free gravitational collapse of the matter. We believe that the general features of the solution obtained this way give a valid indication even for the case that the pressure is not zero, provided that the mass is great enough to cause collapse.
For the solution of this problem, we have found it convenient to follow the earlier work of Tolman ${ }^{2}$ and use another system of coordinates, which are comoving with the matter. After finding a solution, we will introduce a coordinate transformation to put the line element in form (1).

We take a line element of the form :

$$
d s^{2}=d \tau^{2}-e^{\bar{\omega}} d R^{2}-e^{\omega}\left(d \theta^{2}+\sin ^{2} \theta d \varphi^{2}\right) .
$$

Because the coordinates are comoving with the matter and the pressure is zero,

$$
T_{4}^{4}=\rho
$$

and all other components of the energy momentum tensor vanish.

The field equations are :

$$
\begin{gathered}
8 \pi T_{1}{ }^{1}=0=e^{-\omega}-e^{-\bar{\omega}} \frac{\omega^{\prime 2}}{4}+\ddot{\omega}+\frac{3}{4} \dot{\omega}^{2}=0, \\
8 \pi T_{2}{ }^{2}=8 \pi T_{3}{ }^{3}=0=-e^{-\bar{\omega}}\left(\frac{\omega^{\prime \prime}}{2}+\frac{\omega^{\prime 2}}{4}-\frac{\bar{\omega}^{\prime} \omega^{\prime}}{4}\right) \\
+\frac{\ddot{\bar{\omega}}}{2}+\frac{\dot{\bar{\omega}}^{2}}{4}+\frac{\ddot{\omega}}{2}+\frac{\dot{\omega}^{2}}{4}+\frac{\dot{\bar{\omega}} \dot{\omega}}{4}, \\
8 \pi T_{4}{ }^{4}=8 \pi \rho=e^{-\omega}-e^{-\bar{\omega}}\left(\omega^{\prime \prime}+\frac{3}{4} \omega^{\prime 2}-\frac{\bar{\omega}^{\prime} \omega^{\prime}}{2}\right) \\
+\frac{\dot{\omega}^{2}}{4}+\frac{\dot{\bar{\omega} \dot{\omega}}}{2}, \\
8 \pi e^{\bar{\omega}} T_{4}{ }^{1}=-8 \pi T_{1}{ }^{4}=0=\frac{\omega^{\prime} \dot{\omega}}{2}-\frac{\dot{\bar{\omega}} \omega^{\prime}}{2}+\dot{\omega}^{\prime}
\end{gathered}
$$

with primes and dots here and in the following representing differentiation with respect to $R$ and $\tau$, respectively. The integral of Eq. (18) is given by Tolman : ${ }^{3}$

$$
e^{\bar{\omega}}=e^{\omega} \omega^{2} / 4 f^{2}(R)
$$

with $f^{2}(R)$ a positive but otherwise arbitrary function of $R$. We find a sufficiently wide class of solutions if we put $f^{2}(R)=1$.

Substituting (19) in (15) with $f^{2}(R)=1$ we obtain

$$
\ddot{\omega}+\frac{3}{4} \dot{\omega}^{2}=0 .
$$

${ }^{2}$ R. C. Tolman, Proc. Nat. Acad. Sci. 20, 3 (1934).

3 We wish to thank Professor R. C. Tolman and Mr. G. Omer for making this portion of the development available to us, and for helpful discussions. 
The solution of this equation is:

$$
e^{\omega}=(F \tau+G)^{4 / 3},
$$

in which $F$ and $G$ are arbitrary functions of $R$.

The substitution of (19) in (16) gives a result equivalent to (20). Therefore the solution of the field equations is (21).

For the density we obtain from (17), (19), and (21)

$$
8 \pi \rho=4 / 3(\tau+G / F)^{-1}\left(\tau+G^{\prime} / F^{\prime}\right)^{-1} .
$$

There is less real freedom in (21) than is apparent from the two arbitrary functions $F$ and $G$; for taking $R$ a function of a new variable $R^{*}$ the differential equations (15), (17) and (18) will remain of the same form. We may therefore choose

$$
G=R^{\frac{3}{2}} \text {. }
$$

At a particular time, say $\tau$ equal zero, we may assign the density as a function of $R$. Eq. (22) then becomes a first-order differential equation for $F$.

$$
F F^{\prime}=9 \pi R^{2} \rho_{0}(R) .
$$

The solution of this equation contains only one arbitrary constant. We now see that the effect of setting $f^{2}(R)$ equal to one allows us to assign only a one-parameter family of functions for the initial values of $\dot{\rho}_{0}$, whereas in general one should be able to assign the initial values of $\dot{\rho}_{0}$ arbitrarily.

We now take, as a particular case of (24):

$$
F F^{\prime}=\begin{array}{rr}
\text { const. } \times R^{2} ; & \text { const. }>0 ; \\
0 & R<R_{b} \\
0 & R>R_{b} .
\end{array}
$$

A particular solution of this equation is:

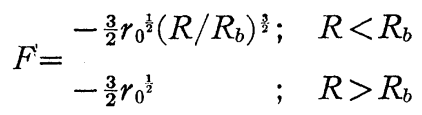

in which the constant $r_{0}$ is introduced for convenience, and is the gravitational radius of the star.

We wish to find a coordinate transformation which will change the line element into form (1). It is clear, by comparison of (1) and (13), that we must take

$$
e^{\omega / 2}=(F \tau+G)^{\frac{2}{3}}=r .
$$

A new variable $t$ which is a function of $\tau$ and $R$ must be introduced so that the $g_{\mu \nu}$ are of the same form as those in Eq. (1). Using the contravarient form of the metric tensor, we find that:

$$
\begin{aligned}
& g^{44}=e^{-\nu}=\dot{t}^{2}-t^{\prime 2} / r^{\prime 2}=\dot{t}^{2}\left(1-\dot{r}^{2}\right), \\
& g^{11}=-e^{-\lambda}=-\left(1-\dot{r}^{2}\right), \\
& g^{14}=0=\dot{t} \dot{r}-t^{\prime} / r^{\prime} .
\end{aligned}
$$

Here (30) is a first-order partial differential equation for $t$. Using the values of $r$ given by (27), and the values of $F$ and $G$ given by (26) and (23) we find:

$\begin{aligned} t^{\prime} / \dot{t}=\dot{r} r^{\prime}= & -\left(r_{0} R\right)^{\frac{1}{2}}\left[R^{\frac{3}{3}}-\frac{3}{2} r_{0}^{\frac{1}{2}} \tau\right]^{-\frac{2}{3}} ; \quad R>R_{b} \\ & -r_{0}^{\frac{1}{2}} R R_{b}{ }^{-\frac{3}{2}}\left[1-\frac{3}{2} r_{0}^{\frac{1}{2}} \tau R_{b}^{-\frac{3}{2}}\right]^{\frac{1}{3}} ; \quad R<R_{b} .\end{aligned}$

The general solution of (31) is:

$$
\begin{array}{r}
t=L(x) \text { for } R>R_{b}, \text { with } x=\frac{2}{3 r_{0}^{\frac{1}{2}}}\left(R^{\frac{3}{2}}-r^{\frac{3}{2}}\right) \\
-2\left(r r_{0}\right)^{\frac{1}{2}}+r_{0} \ln \frac{r^{\frac{1}{2}}+r_{0}^{\frac{1}{2}}}{r^{\frac{1}{2}}-r_{0}^{\frac{1}{2}}} \\
\begin{array}{r}
t=M(y) \text { for } R<R_{b}, \text { with } y=\frac{1}{2}\left[\left(R / R_{b}\right)^{2}-1\right] \\
+R_{b} r / r_{0} R
\end{array}
\end{array}
$$

where $L$ and $M$ are completely arbitrary functions of their arguments.

Outside the star, where $R$ is greater than $R_{b}$, we wish the line element to be of the Schwartzchild form, since we are again neglecting the gravitational effect of any escaping radiation; thus

$$
\begin{aligned}
& e^{\lambda}=\left(1-r_{0} / r\right)^{-1} \\
& e^{\nu}=\left(1-r_{0} / r\right) .
\end{aligned}
$$

This requirement fixes the form of $L$; from (28) we can show that we must take $L(x)=x$, or

$$
t=x .
$$

At the surface of the star, $R$ equal $R_{b}$, we must have $L$ equal to $M$ for all $\tau$. The form of $M$ is determined by this condition to be :

$$
\begin{aligned}
t=M(y)=\frac{2}{3} r_{0}^{-\frac{1}{2}}\left(R_{b}^{\frac{1}{2}}-r_{0}^{\frac{3}{2}} y^{\frac{3}{2}}\right) & \\
& -2 r_{0} y^{\frac{1}{2}}+r_{0} \ln \frac{y^{\frac{1}{2}}+1}{y^{\frac{1}{2}}-1} .
\end{aligned}
$$

Eq. (36), together with (27), defines the transformation from $R, \tau$ to $r$ and $t$, and implicitly, from (28) and (29), the metrical tensor. 
We now wish to find the asymptotic behavior of $e^{\lambda}, e^{\nu}$, and $\tau$ for large values of $t$. When $t$ is large we obtain the approximate relation from Eqs. (36) and (27) :

$$
\begin{aligned}
& t \sim-r_{0} \ln \left\{\frac{1}{2}\left[\left(R / R_{b}\right)^{2}-3\right]\right. \\
& \left.+R_{b} / r_{0}\left(1-3 r_{0}^{\frac{1}{2}} \tau / 2 R_{b}^{2}\right)^{\frac{2}{3}}\right\} .
\end{aligned}
$$

From this relation we see that for a fixed value of $R$ as $t$ tends toward infinity, $\tau$ tends to a finite limit, which increases with $R$. After this time $\tau_{0}$ an observer comoving with the matter would not be able to send a light signal from the star; the cone within which a signal can escape has closed entirely. For a star which has an initial density of one gram per cubic centimeter and a mass of $10^{33}$ grams this time $\tau_{0}$ is about a day.

Substituting (27) and (37) into (28) and (29) we find

$$
\begin{gathered}
e^{-\lambda} \simeq 1-\left(R / R_{b}\right)^{2}\left\{e^{-t / r_{0}}+\frac{1}{2}\left[3-\left(R / R_{b}\right)^{2}\right]\right\}^{-1}, \\
e^{\nu} \simeq e^{\lambda-2 t / r_{0}}\left\{e^{-t / r_{0}}+\frac{1}{2}\left[3-\left(R / R_{b}\right)^{2}\right]\right\} .
\end{gathered}
$$

For $R$ less than $R_{b}, e^{\lambda}$ tends to a finite limit as $t$ tends to infinity. For $R$ equal to $R_{b}, e^{\lambda}$ tends to infinity like $e^{t / r_{0}}$ as $t$ approaches infinity. Where $R$ is less than $R_{b}, e^{\nu}$ tends to zero like $e^{-2 t / r_{0}}$ and where $R$ is equal to $R_{b}, e^{\nu}$ tends to zero like $e^{-t / r_{0}}$.

This quantitative account of the behavior of $e^{\lambda}$ and $e^{\nu}$ can supplement the qualitative discussion given in I. For $\lambda$ tends to a finite limit for $r<r_{0}$ as $t$ approaches infinity, and for $r=r_{0}$ tends to infinity. Also for $r \leqslant r_{0}, \nu$ tends to minus infinity. We expect that this behavior will be realized by all collapsing stars which cannot end in a stable stationary state. Of course, actual stars would collapse more slowly than the example which we studied analytically because of the effect of the pressure of matter, of radiation, and of rotation.

\title{
Formation of Ions in the Cyclotron
}

\author{
ROBERT R. WILSON \\ Radiation Laboratory, Department of Physics, University of California, Berkeley, California
}

(Received July 10, 1939)

\begin{abstract}
Measurements of the initial ionization in a cyclotron, produced by the use of a filament, as a function of the pressure, electron emission, and dee voltage are presented. The amount of ionization is found to be too high to be simply explained by an electron passing between the region between dees only once. A theory is proposed wherein some of the electrons are caught by the changing electric field between the dees and oscillate back and forth many times during a cycle of the dee voltage. Experimental observations which conform to the theory are described.
\end{abstract}

$\mathrm{T}$ $\mathrm{HE}$ manner of formation of the initial ions in a cyclotron is important as it affects the intensity and homogeneity of the high energy beam. Ideally the ions should be formed in a very small region at the center of cyclotron and at the median plane. Livingston, Holloway and Baker $^{1}$ have developed a capillary type of ion source with these characteristics. However, extended filament ion sources are more generally used at present because they give larger circulating currents, and this paper will be concerned

${ }^{1}$ M. S. Livingston, M. G. Holloway and C. P. Baker, Rev. Sci. Inst. 10, 63 (1939). only with an analysis of this type of ion source.

The usual arrangement is for the filament to be located under a shield in which a wide slot is cut to permit the electrons to pass up along the lines of magnetic force between the dees and so ionize by collision the gas in the central region of the cyclotron. A potential difference, hereafter referred to as the emission voltage, between the filament and shield accelerates the electrons from the shielded region.

One is first interested in the magnitude of the ionization between the dees caused by the narrow beam of electrons. This was measured directly 